\title{
Hepatitis B Virus Reactivation during Treatment with Multi-Tyrosine Kinase Inhibitor for Hepatocellular Carcinoma
}

\author{
Satoshi Shiba $^{\mathrm{a}}$ Shunsuke Kondo $^{\mathrm{a}}$ Hideki Ueno $^{\mathrm{a}}$ \\ Chigusa Morizane $^{a}$ Masafumi Ikeda ${ }^{b}$ Takuji Okusaka ${ }^{a}$ \\ ${ }^{a}$ Hepatobiliary and Pancreatic Oncology Division, National Cancer Center \\ Hospital, Tokyo, and ${ }^{\mathrm{b}}$ Hepatobiliary and Pancreatic Oncology Division, \\ National Cancer Center Hospital East, Chiba, Japan
}

\section{Key Words}

Hepatitis B virus reactivation - Hepatocellular carcinoma - Multi-tyrosine kinase inhibitor

\begin{abstract}
Hepatitis B virus (HBV) reactivation is well documented in individuals with cancer who receive certain cytotoxic or immunosuppressive therapies including rituximab treatment. As a general rule, the risk is greatest upon withdrawal of chemotherapy. The risk ranges from approximately 20 to $50 \%$ among HBsAg-positive carriers. A 67-year-old man was diagnosed with inoperable multiple hepatocellular carcinoma accompanied by an increase in alphafetoprotein and protein induced by vitamin $\mathrm{K}$ absence or antagonist II level. Eighteen weeks after starting on the oral multi-tyrosine kinase inhibitor TSU-68, laboratory investigations showed a substantial increase in serum transaminase levels (AST: 302 IU/I; ALT: 324 IU/I) and an elevation of the HBV-DNA level (6.9 log copies $/ \mathrm{ml}$ ). The diagnosis was that the cause of the acute hepatitis was HBV reactivation and we immediately administered entecavir. Two months after the initiation of daily entecavir treatment, laboratory findings showed that the serum levels of transaminases and ALP had improved (AST: 18 IU/I; ALT: 10 IU/I; ALP: 197 $\mathrm{U} / \mathrm{I})$. When the HBV markers were examined 4 months later, they were altered: $\mathrm{HBeAg}$ was negative and $\mathrm{HBeAb}$ was positive. Entecavir treatment was discontinued after 6 months. Although reactivation with rituximab has been reported, reactivation with a tyrosine kinase inhibitor is extremely unusual in a patient who is $\mathrm{HBsAg}$ negative but anti-HBc positive. This is the first report describing HBV reactivation with an increasing HBV-DNA level in a HBsAgnegative/HBcAb-positive/HBsAb-positive patient who was treated with TSU-68 for hepatocellular carcinoma.
\end{abstract}




\section{Introduction}

Hepatitis B virus (HBV) reactivation is well documented in individuals with cancer who receive certain cytotoxic or immunosuppressive therapies including rituximab treatment. As a general rule, the risk is greatest upon withdrawal of chemotherapy. The risk ranges from approximately 20 to $50 \%$ among hepatitis B surface antigen (HBsAg)positive carriers. While any chemotherapy regimen can potentially lead to a reactivation of $\mathrm{HBV}$ replication, the risk may be decreased with steroid-free chemotherapy, implicating the use of glucocorticoids as a risk factor in lymphoma. In patients who are HBsAg negative but hepatitis B core antibody (HBcAb) positive, reactivation with rituximab has been reported.

Hepatocellular carcinoma (HCC) is the fifth most common malignancy worldwide and the third leading cause of cancer-related death [1]. Almost $80 \%$ of all cases are due to an underlying HBV and hepatitis C virus (HCV) infection. For advanced HCC patients, sorafenib, an inhibitor of vascular endothelial growth factor receptor-2 (VEGFR-2), cKit, and raf, has been demonstrated to be active and tolerable [2]. Scientific studies on the molecular pathogenesis of HCC have led to the active development of new drugs. TSU-68 is an orally administered, small-molecule, multiple receptor tyrosine kinase inhibitor that targets VEGFR-2, platelet-derived growth factor receptor, and fibroblast growth factor receptor [3]. Since it is a potent anti-angiogenic agent, TSU-68 is also expected to be effective against HCC.

This is the first report describing HBV reactivation in an $\mathrm{HBsAg}$-negative/ $\mathrm{HBcAb}$ positive/hepatitis B surface antibody (HBsAb)-positive patient who was treated with the oral multi-tyrosine kinase inhibitor (multi-TKI) TSU-68 for HCC.

\section{Case Report}

A 67-year-old man was diagnosed with inoperable multiple HCC accompanied by an increase in alpha-fetoprotein (AFP) and protein induced by vitamin K absence or antagonist II level. Although the patient had neither undergone a blood transfusion nor been tattooed and although he did not drink alcohol or use illicit drugs, he had acquired acute hepatitis with no known cause at 30 years of age and had been treated for chronic hepatitis $C$ with interferon-alpha at 58 years of age. When he was diagnosed with HCC, HBsAg, HCV antibody, and HCV-RNA were negative. HBsAb, HBcAb, and HBVDNA levels were not assessed at the time of diagnosis. His family history of HBV included the following point of interest: his daughter was an HBV carrier. There was no information regarding his late wife's HBV status, as his wife had died almost a decade before.

Transcatheter arterial chemoembolization (TACE) was performed twice upon presentation. Once the patient showed reduction of tumor vascularity on angiography after TACE, he was registered for a phase II clinical trial with the new molecular agent TSU-68, which was administered as an adjuvant chemotherapy after TACE in accordance with the protocol used in the clinical trial. At the beginning of the adjuvant chemotherapy, serum transaminase levels were stabilized within the normal range (aspartate aminotransferase [AST]: $30 \mathrm{IU} / \mathrm{l}$ [normal: $\leq 33 \mathrm{IU} / \mathrm{l}]$; alanine aminotransferase [ALT]: 32 IU/l [normal: $\leq 42 \mathrm{IU} / \mathrm{l}]$ ). Eighteen weeks after starting on the novel treatment, laboratory investigations showed a substantial increase in serum transaminase levels (AST: 302 IU/l; ALT: 324 IU/l; fig. 1). The inhibitor was discontinued immediately. Computed tomography showed that the HCC was not exacerbated, and the serum AFP level was normal. Initiating a treatment with ammonium glycyrrhizate did not ameliorate the hepatocellular injury, and a gradual increase in transaminase levels was noted. The HBV markers were positive for HBsAg, HBcAb, and hepatitis B e antigen (HBeAg), and the quantity of HBV-DNA was $6.9 \mathrm{log}$ copies/ml. HCV antibody and HCV-RNA were negative; the serum markers related to other hepatitis infections such as hepatitis A virus, 
cytomegalovirus, Epstein-Barr virus, and herpes virus were negative. The drug lymphocyte stimulation test yielded a negative result for TSU-68.

Using stored serums, we tested HBsAb, HBcAb, and HBV-DNA levels prior to the initiation of TSU-68; the sample was found to be HBsAb positive and HBcAb positive, with an HBV-DNA level of $2.1 \log$ copies/ml. In addition, molecular analysis showed that the HBV genotype was C, with no HBV mutation in the pre-core or core promoter region. We therefore concluded that the cause of the acute hepatitis was HBV reactivation. We immediately administered entecavir at a dose of $0.5 \mathrm{mg}$ once daily. Two months after starting the daily entecavir treatment, laboratory findings showed that the serum levels of transaminases and alkaline phosphatase (ALP) had improved (AST: 18 IU/l; ALT: 10 IU/l; ALP: 197 U/l). When the HBV markers were examined 4 months later, they were altered: HBeAg was negative and hepatitis B e antibody was positive. Entecavir treatment was discontinued after 6 months, but we continued to observe the patient and carefully monitor his liver function and HCC. One year after commencing the treatment for HBV reactivation, HBsAg was negative, HBsAb was positive, and the quantity of HBV-DNA was undetectable by real-time polymerase chain reaction. After the withdrawal of entecavir, no evidence of increased liver damage or disease progression has been noted during follow-up up to today.

\section{Discussion}

To the best of our knowledge, this is the first report describing $\mathrm{HBV}$ reactivation in an HBsAg-negative/HBcAb-positive/HBsAb-positive patient who was treated with the multi-TKI TSU-68 for HCC in a clinical trial.

Hepatic flare causes an elevation in serum transaminase levels, with the proposed definition of hepatic flare constituting an abrupt increase in serum ALT level to 3-5 times higher than the normal range [4]. HBV flares are usually preceded by an increase in serum HBV-DNA levels. However, because the increase in serum ALT level lags behind the increase in HBV-DNA level, serum HBV-DNA levels may be declining or undetectable when patients with flares are initially evaluated. Thus, HBV reactivation is closely related to the increased quantity of HBV-DNA. In the present patient, who was HBsAg negative, HBsAb positive, and HBcAb positive, serum ALT level increased to more than 7 times the upper limit of the normal range, suggesting a hepatitis flare; the simultaneous increase in the quantity of HBV-DNA allowed us to diagnose HBV reactivation.

$\mathrm{HBV}$ reactivation is known to occur often in individuals with chronic HBV infection. In patients with cancer who are HBsAg positive, especially in those with leukemia and lymphoma, the administration of corticosteroids and rituximab during a hematopoietic cell transplantation therapy has been noted to influence HBV reactivation [5]. Recently, it has been reported that the use of rituximab results in $\mathrm{HBV}$ reactivation in individuals who are HBsAg negative/HBcAb positive or HBsAb positive. This finding suggests that HBsAg-positive patients, as well as HBsAg-negative/HBcAb-positive or HBsAb-positive patients, have a high risk of HBV reactivation [6]. The guidelines issued by the American Association for the Study of Liver Diseases and the American Society of Clinical Oncology Provisional Clinical Opinion recommend that persons receiving cytotoxic or immunosuppressive therapy should be tested for serologic markers of HBV infection so that prophylactic antiviral therapy can be administered to prevent reactivation in HBsAg-positive patients [7].

Apart from rituximab, other drugs that induce HBV reactivation include infliximab, which targets tumor necrosis factor and is used for the treatment of rheumatoid 
arthritis and inflammatory bowel disease [8], and alemtuzumab, which is a humanized monoclonal antibody directed against CD52 that is used for the treatment of chronic lymphocytic leukemia [9]. However, few reports have described small-molecule inhibitors, such as the histone deacetylase inhibitor [10] that is used for T-cell lymphoma and imatinib, which is used for chronic myeloid leukemia. We believe that this report is unique in that it describes HBV reactivation during the administration of a novel multi-TKI in a patient who did not have any hematopoietic disease and who was HBsAg negative, HBsAb positive, and HBcAb positive. In the SHARP trial for advanced HCC, the multi-TKI sorafenib led to liver dysfunction, which resulted in $5 \%$ of the patients discontinuing treatment, but no reports have described an association between such liver dysfunction and HBV reactivation [11]. Although liver dysfunction and an elevation of AST and ALT was noted in $29 \%$ of the patients with HCC in a phase II study of TSU-68 which was also administered to our patient [12], our case highlights the possibility that HBV reactivation may be latent in such patients.

HBV genotype influences clinical outcomes, serum quantitative HBV-DNA levels, fulminant hepatitis, and mutational patterns in the pre-core and core promoter regions [13]. In the patient we presented, the HBV genotype was C, but the serum HBV-DNA level at the time of initiating treatment with TSU-68 was not high, and no HBV mutations were observed in the pre-core or core promoter region. These findings lead us to surmise that HBV genotype directly influences HBV reactivation associated with chemotherapy. On the other hand, it should also be noted that no depletion of neutrophils or lymphocytes occurred in our patient. The inhibition of tyrosine kinase may be relevant to the replication of $\mathrm{HBV}$, although it remains unclear how tyrosine kinase inhibition induces HBV reactivation.

Preemptive therapy with lamivudine for HBsAg-positive patients undergoing chemotherapy reduces the risk of HBV reactivation and HBV-associated morbidity and mortality [14]. Monotherapy with entecavir in adult patients with chronic HBV infection is safe, tolerable, and lowers serum HBV-DNA levels to a greater extent than lamivudine [15]. A clinical trial assessing entecavir as preventive therapy for HBV reactivation associated with rituximab treatment is currently underway. It is unclear whether preventive antiviral therapy for patients receiving multi-TKIs is effective, and the risk of developing $\mathrm{HBV}$ reactivation during such treatment warrants further investigation.

In conclusion, the risk of HBV reactivation associated with multi-TKIs, especially those that inhibit angiogenesis and cell growth, remains unclear. In clinical trials of such new agents, it is difficult to predict the time point at which HBV reactivation occurs following the administration of new molecular agents. Risk classification of chronic HBV infection and preemptive therapy may prevent HBV reactivation and contribute to the development of novel anticancer treatments in this patient population.

\section{Disclosure Statement}

The authors declare that they have no potential conflicts of interest to disclose. 


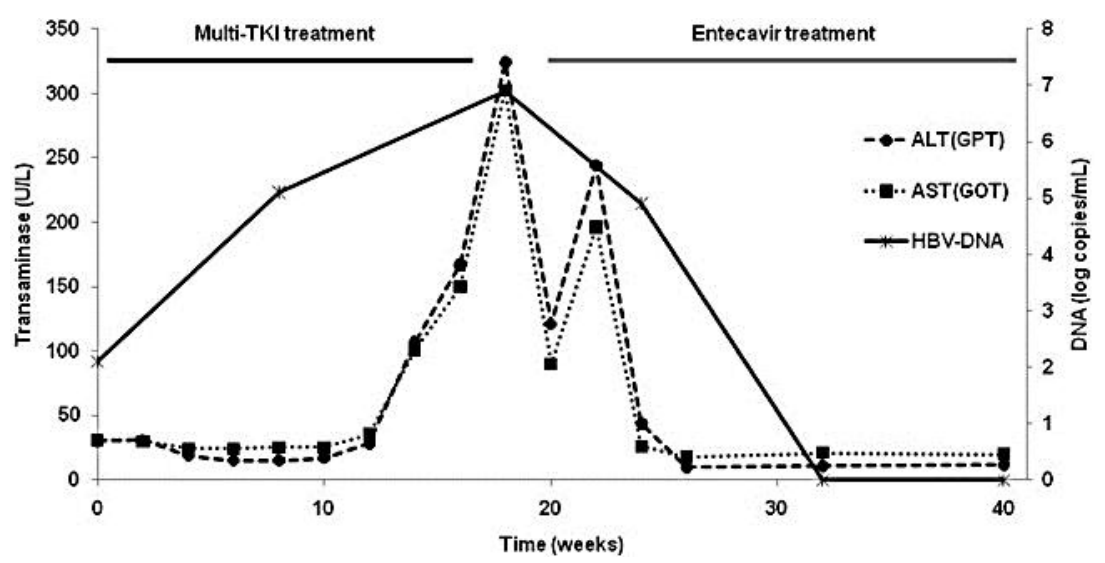

Fig. 1. Changes in serum transaminase levels and HBV-DNA level.

\section{References}

1 Parkin DM, Bray F, Ferlay J, Pisani P: Global cancer statistics, 2002. CA Cancer J Clin 2005;55:74-108.

-2 Llovet JM, Ricci S, Mazzaferro V, Hilgard P, Gane E, Blanc JF, de Oliveira AC, Santoro A, Raoul JL, Forner A, et al: Sorafenib in advanced hepatocellular carcinoma. N Engl J Med 2008;359:378-390.

-3 Laird AD, Vajkoczy P, Shawver LK, Thurnher A, Liang C, Mohammadi M, Schlessinger J, Ullrich A, Hubbard SR, Blake RA, et al: SU6668 is a potent antiangiogenic and antitumor agent that induces regression of established tumors. Cancer Res 2000;60:4152-4160.

$\checkmark 4$ Lok AS, Lai CL, Wu PC, Leung EK, Lam TS: Spontaneous hepatitis B e antigen to antibody seroconversion and reversion in Chinese patients with chronic hepatitis B virus infection. Gastroenterology 1987;92:1839-1843.

$>5$ Cheng AL, Hsiung CA, Su IJ, Chen PJ, Chang MC, Tsao CJ, Kao WY, Uen WC, Hsu CH, Tien HF, et al: Steroidfree chemotherapy decreases risk of hepatitis $B$ virus (HBV) reactivation in HBV-carriers with lymphoma. Hepatology 2003;37:1320-1328.

6 Yeo W, Chan TC, Leung NW, Lam WY, Mo FK, Chu MT, Chan HL, Hui EP, Lei KI, Mok TS, et al: Hepatitis B virus reactivation in lymphoma patients with prior resolved hepatitis B undergoing anticancer therapy with or without rituximab. J Clin Oncol 2009;27:605-611.

7 Lok AS, McMahon BJ: Chronic hepatitis B: update 2009. Hepatology 2009;50:661-662.

-8 Esteve M, Saro C, González-Huix F, Suarez F, Forné M, Viver JM: Chronic hepatitis B reactivation following infliximab therapy in Crohn's disease patients: need for primary prophylaxis. Gut 2004;53:1363-1365.

-9 Iannitto E, Minardi V, Calvaruso G, Mule A, Ammatuna E, Di Trapani R, Ferraro D, Abbadessa V, Craxi A Di Stefano R: Hepatitis B virus reactivation and alemtuzumab therapy. Eur J Haematol 2005;74:254-258.

10 Ritchie D, Piekarz RL, Blombery P, Karai LJ, Pittaluga S, Jaffe ES, Raffeld M, Janik JE, Prince HM, Bates SE: Reactivation of DNA viruses in association with histone deacetylase inhibitor therapy: a case series report. Haematologica 2009;94:1618-1622.

11 Llovet JM, Ricci S, Mazzaferro V, Hilgard P, Gane E, Blanc JF, de Oliveira AC, Santoro A, Raoul JL, Forner A, et al: Sorafenib in advanced hepatocellular carcinoma. N Engl J Med 2008;359:378-390.

12 Kanai F, Yoshida H, Tateishi R, Sato S, Kawabe T, Obi S, Kondo Y, Taniguchi M, Tagawa K, Ikeda M, et al: A phase I/II trial of the oral antiangiogenic agent TSU-68 in patients with advanced hepatocellular carcinoma. Cancer Chemother Pharmacol 2010;67:315-324.

13 Liang TJ, Hasegawa K, Rimon N, Wands JR, Ben-Porath E: A hepatitis B virus mutant associated with an epidemic of fulminant hepatitis. N Engl J Med 1991;324:1705-1709.

14 Loomba R, Rowley A, Wesley R, Liang TJ, Hoofnagle JH, Pucino F, Csako G: Systematic review: the effect of preventive lamivudine on hepatitis B reactivation during chemotherapy. Ann Intern Med 2008;148:519-528.

15 Gish RG, Lok AS, Chang TT, de Man RA, Gadano A, Sollano J, Han KH, Chao YC, Lee SD, Harris M, et al: Entecavir therapy for up to 96 weeks in patients with HBeAg-positive chronic hepatitis B. Gastroenterology 2007;133:1437-1444. 